\title{
Critical Dimensions for counting Lattice Points in Euclidean Annuli
}

\author{
L. Parnovski ${ }^{1}$ and N. Sidorova \\ Department of Mathematics, University College London, Gower St., London WC1E 6BT, UK
}

\begin{abstract}
We study the number of lattice points in $\mathbb{R}^{d}, d \geq 2$, lying inside an annulus as a function of the centre of the annulus. The average number of lattice points there equals the volume of the annulus, and we study the $L_{1}$ and $L_{2}$ norms of the remainder. We say that a dimension is critical, if these norms do not have upper and lower bounds of the same order as the radius goes to infinity. In [6], it was proved that in the case of the ball (instead of an annulus) the critical dimensions are $d \equiv 1 \bmod 4$. We show that the behaviour of the width of an annulus as a function of the radius determines which dimensions are critical now. In particular, if the width is bounded away from zero and infinity, the critical dimensions are $d \equiv 3 \bmod 4$; if the width goes to infinity, but slower than the radius, then all dimensions are critical, and if the width tends to zero as a power of the radius, then there are no critical dimensions.
\end{abstract}

Key words: lattice points

AMS subject classification: 11P21

\section{Introduction}

\subsection{Motivation}

Let $\Gamma$ be a lattice of full rank in $\mathbb{R}^{d}$ with $d \geq 2$; we assume that the volume of the unit cell $\mathcal{O}:=\mathbb{R}^{d} / \Gamma$ is one. For $\mathbf{k} \in \mathcal{O}$ and $\rho>0$ we denote by $N_{\rho}(\mathbf{k})$ the number of lattice points in the open ball $B(\mathbf{k}, \rho)$ centered at $\mathbf{k}$ of radius $\rho$. It is easy to see (and we will show this in the next

\footnotetext{
${ }^{1}$ Corresponding author. E-mail: leonid@math.ucl.ac.uk
} 
section anyway) that

$$
\left\langle N_{\rho}\right\rangle=\omega_{d} \rho^{d},
$$

where we denote $\langle f\rangle:=\int_{\mathcal{O}} f(\mathbf{k}) d \mathbf{k}$, and $\omega_{d}$ is the volume of the unit ball in $\mathbb{R}^{d}$. Many efforts have been spent on studying the upper bounds on the remainder

$$
R_{\rho}(\mathbf{k}):=N_{\rho}(\mathbf{k})-\left\langle N_{\rho}\right\rangle
$$

and estimates with optimal powers of $\rho$ have been obtained in dimensions $d \geq 4$; for $d=2,3$ only non-optimal estimates are known, see [4], [3], and [9] and references therein.

The question of the size of $R_{\rho}$ also plays a very important role in the periodic problems, in particular, in proving the Bethe-Sommerfeld conjecture for periodic Schrödinger operators, see e.g. [8] and [6]. However, the estimates required in periodic problems are of slightly different nature than the classical uniform upper bounds. Namely, let us introduce the following functions:

$$
\sigma_{p}(\rho):=\left\|R_{\rho}\right\|_{p}=\left\langle\left|R_{\rho}\right|^{p}\right\rangle^{1 / p}, \quad p=1,2 ;
$$

the quantity $\sigma_{1}$ can be thought of as an average deviation of $N(\cdot)$ from its average, and $\sigma_{2}(\rho)$ is known as the standard deviation. We will study both upper and lower bounds of these functions, although only lower bounds are needed for the proof of the Bethe-Sommerfeld conjecture. The following theorem was completely proved in [6], although some partial results were obtained in [5] (upper bound) and [8] (lower bound, the case $d \not \equiv 1 \bmod 4$ ).

\section{Theorem 1.}

(a) (Upper bound)

There is $c>0$ such that, for all $\rho$ large enough, one has

$$
\sigma_{1}(\rho) \leq \sigma_{2}(\rho) \leq c \rho^{\frac{d-1}{2}}
$$

(b) (Lower bound)

Suppose $d \not \equiv 1 \bmod 4$. There is $c>0$ such that, for all $\rho$ large enough, one has

$$
\sigma_{1}(\rho) \geq c \rho^{\frac{d-1}{2}} .
$$

Suppose $d \equiv 1 \bmod 4$ and $\varepsilon>0$. There is $c>0$ such that, for all $\rho$ large enough, one has

$$
\sigma_{1}(\rho) \geq c \rho^{\frac{d-1}{2}-\varepsilon} .
$$

(c) (Exactness of the lower bound)

Suppose $d \equiv 1 \bmod 4$ and $\varepsilon>0$. There exists a sequence $\rho_{n} \rightarrow \infty$ such that

$$
\sigma_{2}\left(\rho_{n}\right) \leq \rho_{n^{\frac{d-1}{2}}}\left(\ln \rho_{n}\right)^{(-1+\epsilon) / d}
$$


Using these estimates, one can prove Bethe-Sommerfeld conjecture for Schrödinger operators in dimensions 2, 3, 4 and for some other periodic operators, see [6] and [7] for details; however, these estimates are not sufficient to prove the conjecture for Schrödinger operators in dimensions $d \geq 5$.

An immediate observation one can make from Theorem 1 is the following: if $d \not \equiv 1 \bmod 4$, then both $\sigma_{1}$ and $\sigma_{2}$ have upper and lower bounds with the same power of $\rho$, whereas if $d \equiv$ $1 \bmod 4$, such bounds do not exist. This makes it natural to call the cases $d \equiv 1 \bmod 4$ the critical dimensions. The question we want to ask is whether there are different setups where (for similar problems) the critical dimensions take other values.

\subsection{Results}

This paper deals with the situation when instead of counting lattice points inside the ball, we count lattice points inside the annuli. Thus, we introduce two parameters: $\rho$ (the radius of the annulus) and $\eta=\eta(\rho)$ (half-width of the annulus) which we assume to be a continuous function of $\rho$ with $\eta<\rho$. We denote by $N_{\rho, \eta}(\mathbf{k})$ the number of lattice points in the annulus $B(\mathbf{k}, \rho, \eta):=$ $B(\mathbf{k}, \rho+\eta) \backslash B(\mathbf{k}, \rho-\eta)$. Similar to the case of the ball, we have

$$
\left\langle N_{\rho, \eta}\right\rangle=\omega_{d}\left[(\rho+\eta)^{d}-(\rho-\eta)^{d}\right]
$$

and we define

$$
R_{\rho, \eta}(\mathbf{k}):=N_{\rho, \eta}(\mathbf{k})-\left\langle N_{\rho, \eta}\right\rangle .
$$

The purpose of this paper is to find estimates of the following averages of $R$ :

$$
\sigma_{p}(\rho, \eta):=|| R_{\rho, \eta} \|_{p}=\left\langle\left|R_{\rho, \eta}\right|^{p}\right\rangle^{1 / p}, \quad p=1,2,
$$

and, in particular, to establish which dimensions are critical. The answer will depend on how exactly $\eta$ depends on $\rho$. There are four essentially different regimes of the behaviour of $\eta$ :

(i) $\eta=c \rho$;

(ii) $\eta \rightarrow \infty$ but $\eta / \rho \rightarrow 0$;

(iii) $\eta \asymp 1$ (that is, $\eta$ is bounded away from zero and infinity);

(iv) $\eta \rightarrow 0$.

The first regime is the simplest one: here the answer is exactly the same as it is in the case of the ball, namely, critical dimensions are $d \equiv 1 \bmod 4$. The proof of this fact is also very similar to the case of the ball, and we will not discuss it in detail. The other regimes are much more interesting. In particular, in the case (ii) all dimensions are critical, and in the case (iii) critical dimensions are $d \equiv 3 \bmod 4$. The case (iv) is the most difficult one; we can only treat the case $p=2$, and the answer depends on how quickly $\eta$ tends to zero. If $\eta$ tends to zero slower than any power of $\rho$, then the situation is similar to the case (iii), that is, critical dimensions are $d \equiv 3 \bmod 4$. If, on the 
other hand, $\eta \asymp \rho^{-\gamma}$ with some positive $\gamma$, then there are no critical dimensions. We would like to mention here that in the case $d=2$ and $\eta=c \rho^{-1}$, a much more detailed information about the behaviour of $\sigma_{2}$ was obtained in [2]. We will formulate the precise theorems in the sections where we discuss the corresponding regimes.

The paper is organized as follows. In Section 2 we prove some technical statements which are relevant to several regimes of $\eta$ simultaneously. In Section 3, 4, and 5 we discuss the regimes (ii), (iii), and (iv), respectively.

Acknowledgement. We are very grateful to Richard Hill who took part in the early stages of this work, but withdrew later. Some of the results of this paper were obtained with his participation.

\subsection{Outline of the proofs}

Denote

$$
\theta:=\pi \frac{d+1}{4}
$$

Denote by $\Gamma^{*}=\left\{\mathbf{b} \in \mathbb{R}^{d}: e^{i\langle\mathbf{a}, \mathbf{b}\rangle} \in 2 \pi \mathbb{Z}\right.$ for all $\left.\mathbf{a} \in \Gamma\right\}$ the lattice (analytically) dual to $\Gamma$ and by $\left\{\mathbf{e}_{j}\right\}$ any fixed basis of $\Gamma^{*}$. We also put $\mathcal{O}^{*}:=\mathbb{R}^{d} / \Gamma^{*}$. For any vector $\mathbf{b} \in \mathbb{R}^{d}$ we denote by $b=|\mathbf{b}|$ its Euclidean norm. For any integrable function $f: \mathcal{O} \rightarrow \mathbb{C}$ we denote by

$$
\hat{f}(\mathbf{b})=\int_{\mathcal{O}} f(\mathbf{k}) e^{i \mathbf{b k}} d \mathbf{k}, \quad \mathbf{b} \in \Gamma^{*}
$$

its Fourier coeffiients.

First, we observe that $\sigma_{1}(\rho, \eta)$ and $\sigma_{2}(\rho, \eta)$ can be estimated in terms of the Fourier coefficients as

$$
\left|\hat{R}_{\rho, \eta}(\mathbf{b})\right| \leq \sigma_{1}(\rho, \eta) \leq \sigma_{2}(\rho, \eta)=\left(\sum_{\mathbf{b} \in \Gamma^{*}} \hat{R}_{\rho, \eta}^{2}(\mathbf{b})\right)^{\frac{1}{2}} .
$$

Then we compute the values of the Fourier coefficients in terms of the Bessel functions of the first kind and, using our knowledge about their asymptotic behaviour at infinity, compute the asymptotics of the Fourier coefficients

$$
\hat{R}_{\rho, \eta(\rho)}(\mathbf{b})=c \rho^{\frac{d-1}{2}} b^{-\frac{d+1}{2}} \sin (b \eta(\rho)) \sin (b \rho-\theta)+\eta(\rho) \rho^{\frac{d-3}{2}} b^{-\frac{d+1}{2}} O(1)
$$

as $\eta / \rho \rightarrow 0$. Now the upper bound for $\sigma_{2}(\rho, \eta)$ can be easily obtained either from Parseval's identity (for the regime $\eta(\rho) \nrightarrow 0$ ) or just simply from the upper bounds for the balls given in Theorem 1.

It is much more difficult to get lower bounds for $\sigma_{1}(\rho, \eta)$. In the regimes when $\eta \nrightarrow 0$ the estimate on the left hand side of (1.5) is good enough, but it will require lots of efforts to find an element $\mathbf{b}$ of the dual lattice $\Gamma^{*}$ giving roughly the same contribution as all other terms together. This is due to the trigonometric term in (1.6) which should be kept away from zero. In some regimes and dimensions, for any $\rho$ we can find such $\mathbf{b}$ in a bounded region around zero. This 
provides a lower bound which is the same as the upper bound and so such dimensions are noncritical for the corresponding regimes. However, sometimes a suitable $\mathbf{b}$ can only be found at a distance from the origin tending to infinity together with $\rho$. This reduces the lower bound by an arbitrarily small power of $\rho$ (due to the multiple $b^{-\frac{d+1}{2}}$ in (1.6)) and so creates a gap between the lower and upper bound. This corresponds to critical dimensions.

For every critical dimension, we show that the gap between the lower and upper bound is not an artifact in our proof, that is, that there are no lower and upper bounds with the same powers of $\rho$. This is done using pigeonhole principle to find a sequence of specific $\rho_{n}$ such that exceptionally many of the Fourier coefficients are small. This gives a sequence $\rho_{n}$, for which $\sigma_{2}\left(\rho_{n}, \eta\left(\rho_{n}\right)\right)$ is essentially smaller then the upper bound.

In the regime $\eta \rightarrow 0$, the estimate on the left hand side of (1.5) is too weak, as a single Fourier coefficient does not give a significant contribution anymore. Because of this, we are not able to find a suitable lower bound for $\sigma_{1}(\rho, \eta)$ and restrict our study to $\sigma_{2}(\rho, \eta)$. For the latter, we are able to identify a sufficient number of Fourier coefficients contributing to its value, and find a lower bound using Parseval's identity. Similarly to the other regimes, sometimes the properties of the conributing elements $\mathbf{b}$ are good enough to provide a lower bound which is the same as the upper bound, and sometimes they are not. This corresponds to non-critical and critical dimensions.

\section{Preliminary results}

In this section we prove some technical statements which will be used later and which are relevant to several regimes of $\eta$ simultaneously. In Lemma 2 we give simple upper and lower bounds for the norms $\left\|R_{\rho, \eta}\right\|_{1}$ and $\left\|R_{\rho, \eta}\right\|_{2}$. Since the lower bound is in terms of the Fourier coefficients and as we will later use Parseval's identity to further estimate $\left\|R_{\rho, \eta}\right\|_{2}$, in Lemma 3 we compute the asymptotics of the Fourier coefficients in terms of the Bessel functions. In Lemma 4 we study two explicit families of functions which are closely related to computing the lengths of elements of the lattice. Finally, Lemma 5 is one of the most important tools to prove main results of the paper. It guarantees that the leading term in the asymptotics of the Fourier coefficients found in Lemma 3 can be kept away from zero despite the oscillating trigonometric term.

Let $B(\rho)$ denote the open ball of radius $\rho>0$ in $\mathbb{R}^{d}$ centered at the origin and let $\chi(\cdot, \rho)$ be its characteristic function. Then, for $\mathbf{k} \in \mathcal{O}$ and $\rho>0$,

$$
N_{\rho}(\mathbf{k})=\sum_{\mathbf{m} \in \Gamma} \chi(\mathbf{m}-\mathbf{k}, \rho)
$$

and so

$$
\left\langle N_{\rho}\right\rangle=\int_{\mathcal{O}} \sum_{\mathbf{m} \in \Gamma} \chi(\mathbf{m}-\mathbf{k}, \rho) d \mathbf{k}=\int_{\mathbb{R}^{d}} \chi(\mathbf{k}, \rho) d \mathbf{k}=\operatorname{vol}(B(\rho))=\omega_{d} \rho^{d},
$$

where $\omega_{d}$ is the volume of the unit ball in $\mathbb{R}^{d}$. Obviously, $N_{\rho, \eta}=N_{\rho+\eta}-N_{\rho-\eta}$ and hence

$$
\left\langle N_{\rho, \eta}\right\rangle=\left\langle N_{\rho+\eta}\right\rangle-\left\langle N_{\rho-\eta}\right\rangle=\operatorname{vol}(B(\rho+\eta) \backslash B(\rho-\eta))=\omega_{d}\left[(\rho+\eta)^{d}-(\rho-\eta)^{d}\right] .
$$


So the remainder term $R_{\rho, \eta}$ can be written as

$$
R_{\rho, \eta}=R_{\rho+\eta}-R_{\rho-\eta}
$$

Lemma 2. There is a constant $c>0$ such that, for all $0<\eta<\rho$ and $\mathbf{b} \in \Gamma^{*}$

$$
\left|\hat{R}_{\rho, \eta}(\mathbf{b})\right| \leq \sigma_{1}(\rho, \eta) \leq \sigma_{2}(\rho, \eta)<c \rho^{\frac{d-1}{2}}
$$

Proof. The lower bound follows from

$$
\left\|R_{\rho, \eta}\right\|_{1}=\int_{\mathcal{O}}\left|R_{\rho, \eta}(\mathbf{k})\right| d \mathbf{k} \geq\left|\int_{\mathcal{O}} R_{\rho, \eta}(\mathbf{k}) e^{i \mathbf{b k}} d \mathbf{k}\right|=\left|\hat{R}_{\rho, \eta}(\mathbf{b})\right| .
$$

To prove the upper bound, observe that according to [6, Th. 3.1] there is a constant $c_{1}$ such that

$$
\left\|R_{\rho}\right\|_{2}<c_{1} \rho^{\frac{d-1}{2}} \text { for all } \rho>0 .
$$

Although it has been formulated there only for $\rho$ large enough, it is easy to see that it is actually true for all $\rho>0$ because of the continuity of $\left\|R_{\rho}\right\|_{2}$ in $\rho$ and since

$$
\left\|R_{\rho}\right\|_{2}^{2} \leq \operatorname{vol}(B(\rho))(1-\operatorname{vol}(B(\rho))) \leq \omega_{d} \rho^{d-1}
$$

for $\rho$ small enough.

Using $\eta<\rho$, we obtain

$$
\begin{aligned}
\left\|R_{\rho, \eta}\right\|_{1} & \leq\left\|R_{\rho, \eta}\right\|_{2}=\left\|R_{\rho+\eta}-R_{\rho-\eta}\right\|_{2} \leq\left\|R_{\rho+\eta}\right\|_{2}+\left\|R_{\rho-\eta}\right\|_{2} \\
& <2 c_{1}(\rho+\eta)^{\frac{d-1}{2}}<c_{1} 2^{\frac{d+1}{2}} \rho^{\frac{d-1}{2}} \leq c \rho^{\frac{d-1}{2}}
\end{aligned}
$$

for some $c>0$.

Lemma 3. For any $\mathbf{b} \in \Gamma^{*}$,

$$
\hat{R}_{\rho, \eta}(\mathbf{b})= \begin{cases}\left(\frac{2 \pi(\rho+\eta)}{b}\right)^{d / 2} J_{d / 2}(b(\rho+\eta))-\left(\frac{2 \pi(\rho-\eta)}{b}\right)^{d / 2} J_{d / 2}(b(\rho-\eta)) & \text { if } \mathbf{b} \neq \mathbf{0} \\ 0 & \text { if } \mathbf{b}=\mathbf{0}\end{cases}
$$

where $J_{\nu}$ denotes the Bessel function of the first kind.

If $\eta(\rho) / \rho \rightarrow 0$ as $\rho \rightarrow \infty$, then

$$
\hat{R}_{\rho, \eta(\rho)}(\mathbf{b})=-2 \sqrt{\frac{2}{\pi}}(2 \pi)^{\frac{d}{2}} \rho^{\frac{d-1}{2}} b^{-\frac{d+1}{2}} \sin (b \eta(\rho)) \sin (b \rho-\theta)+\eta(\rho) \rho^{\frac{d-3}{2}} b^{-\frac{d+1}{2}} O(1)
$$

uniformly in $\mathbf{b} \in \Gamma^{*} \backslash\{0\}$. 
Proof. Repeating the computations from [6] we have, for all $\mathbf{b} \in \Gamma^{*} \backslash\{0\}$,

$$
\hat{N}_{\rho}(\mathbf{b})=\int_{\mathcal{O}} N_{\rho}(\mathbf{k}) e^{i \mathbf{b} \mathbf{k}} d \mathbf{k}=\int_{\mathcal{O}} \sum_{\mathbf{m} \in \Gamma} \chi(\mathbf{m}-\mathbf{k}, \rho) e^{i \mathbf{b k}} d \mathbf{k}=\int_{|\mathbf{k}|<\rho} e^{i \mathbf{b k}} d \mathbf{k}=\left(\frac{2 \pi \rho}{b}\right)^{d / 2} J_{d / 2}(b \rho) .
$$

Similarly, $\hat{N}_{\rho}(0)=\omega_{d} \rho^{d}$. Hence

$$
\hat{R}_{\rho}(\mathbf{b})= \begin{cases}\left(\frac{2 \pi \rho}{b}\right)^{d / 2} J_{d / 2}(b \rho) & \text { if } \mathbf{b} \neq \mathbf{0} \\ 0 & \text { if } \mathbf{b}=\mathbf{0}\end{cases}
$$

Now (2.1) follows from $\hat{R}_{\rho, \eta}(\mathbf{b})=\hat{R}_{\rho+\eta}(\mathbf{b})-\hat{R}_{\rho-\eta}(\mathbf{b})$.

Suppose now that $\lim _{\rho \rightarrow \infty} \eta(\rho) / \rho=0$. The Bessel function $J_{d / 2}$ has the following asymptotics as $x \rightarrow \infty$ (see formula (4.8.5) of [1])

$$
J_{d / 2}(x) \propto \sqrt{\frac{2}{\pi x}}\left(\cos (x-\theta) \sum_{n=0}^{\infty}(-1)^{n} a_{2 n} x^{-2 n}-\sin (x-\theta) \sum_{n=0}^{\infty}(-1)^{n} a_{2 n+1} x^{-2 n-1}\right)
$$

with $a_{0}=1$ and some real coefficients $a_{k}, k \geq 1$. The symbol $\propto$ here means that this asymptotic is true when truncated after an arbitrary power $x^{-k}$ of $x$, with the error of order $O\left(x^{-k-1}\right)$. Moreover, this asymptotics can be differentiated termwise.

Let

$$
\begin{aligned}
\widetilde{J}_{d / 2}(x) & =J_{d / 2}(x) \sqrt{\frac{\pi x}{2}}-\cos (x-\theta) \\
& \propto \cos (x-\theta) \sum_{n=1}^{\infty}(-1)^{n} a_{2 n} x^{-2 n}-\sin (x-\theta) \sum_{n=0}^{\infty}(-1)^{n} a_{2 n+1} x^{-2 n-1} .
\end{aligned}
$$

Then $\widetilde{J}_{d / 2}(x)=O\left(x^{-1}\right)$ and $\widetilde{J}_{d / 2}^{\prime}(x)=O\left(x^{-1}\right)$ as $x \rightarrow \infty$. Using (2.1) we obtain for $\mathbf{b} \in \Gamma^{*} \backslash\{0\}$,

$$
\begin{aligned}
\hat{R}_{\rho, \eta(\rho)}(\mathbf{b}) & =\left(\frac{2 \pi(\rho+\eta(\rho))}{b}\right)^{d / 2} J_{d / 2}(b(\rho+\eta(\rho)))-\left(\frac{2 \pi(\rho-\eta(\rho))}{b}\right)^{d / 2} J_{d / 2}(b(\rho-\eta(\rho))) \\
& =\sqrt{\frac{2}{\pi}}(2 \pi)^{\frac{d}{2}}(\rho+\eta(\rho))^{\frac{d-1}{2}} b^{-\frac{d+1}{2}}\left(\widetilde{J}_{d / 2}(b(\rho+\eta(\rho)))+\cos (b(\rho+\eta(\rho))-\theta)\right) \\
& -\sqrt{\frac{2}{\pi}}(2 \pi)^{\frac{d}{2}}(\rho-\eta(\rho))^{\frac{d-1}{2}} b^{-\frac{d+1}{2}}\left(\widetilde{J}_{d / 2}(b(\rho-\eta(\rho)))+\cos (b(\rho-\eta(\rho))-\theta)\right) \\
= & \sqrt{\frac{2}{\pi}}(2 \pi)^{\frac{d}{2}} \rho^{\frac{d-1}{2}} b^{-\frac{d+1}{2}}(\cos (b(\rho+\eta(\rho))-\theta)-\cos (b(\rho-\eta(\rho))-\theta) \\
& \left.+\widetilde{J}_{d / 2}(b(\rho+\eta(\rho)))-\widetilde{J}_{d / 2}(b(\rho-\eta(\rho)))+O(\eta(\rho) / \rho)\right) \\
= & \sqrt{\frac{2}{\pi}(2 \pi)^{\frac{d}{2}} \rho^{\frac{d-1}{2}} b^{-\frac{d+1}{2}}\left(-2 \sin (b \eta(\rho)) \sin (b \rho-\theta)+2 b \eta(\rho)+\widetilde{J}_{d / 2}^{\prime}(\xi(b, \rho))\right.} \\
& +O(\eta(\rho) / \rho))
\end{aligned}
$$


where $\xi(b, \rho) \in\left(b\left(\rho-\eta(\rho), b(\rho+\eta(\rho))\right.\right.$ and $O(\cdot)$ is uniform in $\mathbf{b}$. Using the asymptotics for $\widetilde{J}_{d / 2}^{\prime}$ we obtain $\widetilde{J}_{d / 2}^{\prime}(\xi(b, \rho))=(b \rho)^{-1} O(1)$ uniformly in $\mathbf{b}$, which completes the proof.

Lemma 4. (1) Let $u>0$ and $w$ be such that $|w|<u$. Let $m \in \mathbb{N}$ and $I>0$. For each $t>0$, $x \in[0, I]$ and $^{2} k \in \mathbb{Z} \cap[1, m]$ denote

$$
f_{k, x}(t)=\sqrt{(1+x t)^{2}+k^{2} t^{2} u^{2}+2 k t(1+x t) w} .
$$

Then there is $t_{f}>0$ such that

$$
f_{k, x}(t)=\sum_{n=0}^{\infty} a_{n}(k, x) t^{n}
$$

on $\left[0, t_{f}\right]$, where $a_{0}(k, x)=1, a_{1}(k, x)=x+k w$, and $a_{n}(k, \cdot)$ is a polynomial in $x$ of degree exactly $n-2$ for all $n \geq 2$.

(2) Let $v \in \mathbb{R}$ and let $m \in \mathbb{N}$. For each $t>0$ and $x \in[-1,1]$, denote

$$
g_{x}(t)=\sqrt{1+2 t x+t^{2}} .
$$

Then there is $t_{g}>0$ such that

$$
g_{x}(t)=\sum_{n=0}^{\infty} b_{n}(x) t^{n}
$$

uniformly on $[-1,1] \times\left[0, t_{g}\right]$, where $b_{n}(\cdot)$ is a polynomial in $x$ of degree exactly $n$ for all $n \geq 0$.

Proof. (1) First, let us show that the functions $f_{k, x}$ (considered as functions on the complex plane) have no singularities in the open ball of radius $1 /(I+m u)$ around zero (which would imply the choice of $t_{f}$ ). Indeed, observe that, since $|w|<u$,

$$
f_{k, x}^{2}(t)=\left(x^{2}+k^{2} u^{2}+2 k x w\right) t^{2}+2(x+k w) t+1
$$

has two complex roots

$$
t_{1,2}=\frac{-x-k w \pm i k \sqrt{u^{2}-w^{2}}}{x^{2}+k^{2} u^{2}+2 k x w}
$$

and so

$$
\left|t_{1,2}\right|=\left(x^{2}+k^{2} u^{2}+2 k x w\right)^{-1 / 2} \geq 1 /(x+k u) \geq 1 /(I+m u) .
$$

Further, using $a_{n}(k, x)=f_{k, x}^{(n)}(0) / n$ ! and differentiating (2.5) we obtain the required formulas for $a_{0}$ and $a_{1}$ as well as $a_{2}(k, x)=k^{2}\left(u^{2}-w^{2}\right) / 2$. Continuing for $n \geq 3$ we get

$$
2 f_{k, x} f_{k, x}^{(n)}+2 n f_{k, x}^{(1)} f_{k, x}^{(n-1)}+\sum_{j=2}^{n-2}\left(\begin{array}{c}
n \\
j
\end{array}\right) f_{k, x}^{(j)} f_{k, x}^{(n-j)}=0 .
$$

\footnotetext{
${ }^{2}$ There was a mistake here: $k$ should not be equal to zero, othervise the polynomials degenerate.
} 
Evaluating it at zero and using induction we obtain that $f_{k, x}^{(1)}(0) f_{k, x}^{(n-1)}(0)$ is a polynomial of degree $n-2$ and $f_{k, x}^{(j)}(0) f_{k, x}^{(n-j)}(0)$ are polynomials of degree $n-4$, which implies that $f_{k, x}^{(n)}(0)$ is a polynomial of degree $n-2$ and so is $a_{n}(k, \cdot)$.

(2) First, let us show that the functions $g_{k, x}$ (considered as functions on the complex plane) have no singularities in the open ball of radius 1 around zero (which would imply the choice of $t_{g}$ ). Indeed, since $|x|<1$ it has two zeroes $t_{1,2}=-x \pm i \sqrt{1-x^{2}}$, which satisfy $\left|t_{1,2}\right|=1$.

Further, using $b_{n}(x)=g_{x}^{(n)}(0) / n$ ! and differentiating $g_{x}^{2}(t)=1+2 t x+t^{2}$ we obtain $b_{0}(x)=1$, $b_{1}(x)=x$ and $b_{2}(x)=\left(1-x^{2}\right) / 2$. Continuing for $n \geq 3$, we get

$$
2 g_{x} g_{x}^{(n)}+\sum_{j=1}^{n-1}\left(\begin{array}{c}
n \\
j
\end{array}\right) g_{x}^{(j)} g_{x}^{(n-j)}=0 .
$$

Evaluating it at zero and using induction we obtain that $g_{x}^{(j)}(0) g_{x}^{(n-j)}(0)$ are polynomials of degree $n$, which implies that $g_{x}^{(n)}(0)$ is a polynomial of degree at most $n$.

To prove that the degree of $g_{x}^{(n)}(0)$ (and so of $b_{n}$ ) is exactly equal to $n$, denote its coefficient at $x^{n}$ by $p_{n}$. Then $p_{0}=1, p_{1}=1, p_{2}=-1$ and (2.6) implies the following recurrent formula for $n \geq 3$

$$
p_{n}=-\frac{1}{2} \sum_{j=1}^{n-1}\left(\begin{array}{l}
n \\
j
\end{array}\right) p_{j} p_{n-j} .
$$

It can be easily seen by induction that $p_{n}=(-1)^{n+1}\left|p_{n}\right|$ for $n \geq 1$ and $p_{n} \neq 0$ as $p_{j} p_{n-j}=$ $(-1)^{n}\left|p_{j}\right|\left|p_{n-j}\right|$ for all $1 \leq j \leq n-1$.

It remains to prove that the series representing $g_{x}(t)$ converges uniformly in $x$ and $t$. Let $q_{n}, n \in \mathbb{N} \cup\{0\}$ be Catalan numbers, that is, $q_{0}=1$ and $q_{n+1}=\sum_{j=0}^{n} q_{j} q_{n-j}$ for $n \geq 0$. Let us prove that $\left|g_{x}^{(n)}(0)\right| \leq 2 n ! q_{n-1}$ for all $n \in \mathbb{N}$. For $n=1$ we have $\left|g_{x}^{(1)}(0)\right|=|x| \leq 1$ and for $n=2$ we have $\left|g_{x}^{(2)}(0)\right|=1-x^{2} \leq 1$, which imply the required formulas. For $n \geq 3$, it follows inductively from (2.6) as

$$
\left|g_{x}^{(n)}(0)\right| \leq \frac{1}{2} \sum_{j=1}^{n-1} \frac{n !}{j !(n-j) !}\left|g_{x}^{(j)}(0)\right|\left|g_{x}^{(n-j)}(0)\right|=2 n ! \sum_{i=0}^{n-2} q_{i} q_{n-2-i}=2 n ! q_{n-1} .
$$

This implies $\left|b_{n}(x) t^{n}\right| \leq 2 q_{n-1} t_{g}^{n}$. Since the radius of convergence of the series $\sum_{n=1}^{\infty} q_{n} t^{n}$ is $1 / 4$, the series (2.4) converges uniformy in $x$ and $t$ once $t_{g}<1 / 4$.

For each $x \in \mathbb{R}$ we denote by $\omega[x]$ the distance from $x / \pi$ to the nearest integer. Observe that $\omega$ satisfies the triangle inequality and $\omega[n x] \leq n \omega[x]$ for any $x \in \mathbb{R}$ and $n \in \mathbb{Z}$.

Lemma 5. Suppose $\eta$ is such that

$$
\liminf _{\rho \rightarrow \infty} \frac{\log \eta(\rho)}{\log \rho} \geq 0
$$


Then for any $\varepsilon>0$ there exists $\alpha \in(0,1 / 2)$ such that for any $\rho$ large enough one can find an element $\mathbf{b}(\rho) \in \Gamma^{*}$ with the properties $b(\rho) \leq \rho^{\varepsilon}, \omega[b(\rho) \rho-\theta] \geq \alpha$, and $\omega[b(\rho) \eta(\rho)] \geq \alpha$.

Proof. Let $\varepsilon>0$ be given. Without loss of generality we assume $\varepsilon<1$. Let $m \in \mathbb{N}$ be such that $0<\frac{1}{m-2}<\varepsilon / 8, L=2 m$, and $I=L m+1$.

Observe that the inequality $\omega[b(\rho) \rho-\theta] \geq \alpha$ follows from the inequality $\omega[4 b(\rho) \rho] \geq 4 \alpha$ since $\theta \in(\pi / 4) \mathbb{Z}$. We will prove the statement of the lemma with the latter inequality instead of the former.

Step 1. We start by slightly generalising the proof of Lemma 3.3 from [6]. Namely, we will find $\alpha \in(0,1 / 6)$, an integer valued function $n(\rho)$ satisfying $n(\rho) \asymp \rho^{\frac{1}{m-1}}$ and integer-valued functions $k_{i}(\rho), 0 \leq i \leq I$, taking values between 1 and $m$ such that all elements

$$
\mathbf{b}_{i}(\rho)=(n(\rho)+i) \mathbf{e}_{1}+k_{i}(\rho) \mathbf{e}_{2}, \quad 0 \leq i \leq I,
$$

satisfy $b_{i}(\rho) \leq \rho^{\varepsilon / 3}, \omega\left[4 b_{i}(\rho) \rho\right] \geq 8 \alpha$.

For any $n \in \mathbb{N}$ and $k \in \mathbb{Z} \cap[1, m]$, the length of the vector $n \mathbf{e}_{1}+k \mathbf{e}_{2}$ is given by

$$
B_{n}(k)=\sqrt{n^{2}\left|\mathbf{e}_{1}\right|^{2}+k^{2}\left|\mathbf{e}_{2}\right|^{2}+2 n k\left\langle\mathbf{e}_{1}, \mathbf{e}_{2}\right\rangle} .
$$

Denote $B_{n}^{(1)}(k)=B_{n}(k+1)-B_{n}(k), k \in \mathbb{Z} \cap[1, m-1]$, and, for all $2 \leq i \leq m-1$, $B_{n}^{(i)}(k)=B_{n}^{(i-1)}(k+1)-B_{n}^{(i-1)}(k), k \in[1, m-i]$. Slightly adjusting the proof of Lemma 3.3 from [6], we obtain that

$$
B_{n}^{(m-1)}(1)=A n^{2-m}\left(1+O\left(n^{-1}\right)\right)
$$

where $A \neq 0$. Define

$$
n(\rho)=\left\lfloor\left(8|A| \pi^{-1} \rho\right)^{\frac{1}{m-2}}\right\rfloor,
$$

where $\lfloor\cdot\rfloor$ denotes taking the integer part. Then, for each $0 \leq i \leq I$,

$$
\left|4 B_{n(\rho)+i}^{(m-1)}(1) \rho\right|=\pi / 2+o(1)
$$

as $\rho \rightarrow \infty$, so that

$$
\omega\left[4 B_{n(\rho)+i}^{(m-1)}(1) \rho\right]=1 / 2+o(1) .
$$

Now let $\alpha$ be such that $2^{m+2} \alpha<1 / 4$, which in particular implies $\alpha \in(0,1 / 6)$. For each $i$, if $\omega\left[4 B_{n(\rho)+i}(k) \rho\right]<8 \alpha$ for all $k \in \mathbb{Z} \cap[1, m]$ then $\omega\left[4 B_{n(\rho)+i}^{(m-1)}(1) \rho\right]<2^{m-1} 8 \alpha<1 / 4$, which contradicts (2.10) for $\rho$ large enough. Hence for each $i$ and $\rho$ there is $k_{i}(\rho) \in \mathbb{Z} \cap[1, m]$ such that $\omega\left[4 B_{n(\rho)+i}\left(k_{i}(\rho)\right) \rho\right] \geq 8 \alpha$ and so the elements $\mathbf{b}_{i}$ defined in (2.8) satisfy $\omega\left[4 b_{i}(\rho) \rho\right] \geq 8 \alpha$. The estimate $b_{i}(\rho) \leq \rho^{\varepsilon / 3}$ follows from $\frac{1}{m-2}<\varepsilon / 8<\varepsilon / 3$.

Step 2. Denote $u=\left|\mathbf{e}_{2}\right| /\left|\mathbf{e}_{1}\right|$ and $w=\left\langle\mathbf{e}_{1}, \mathbf{e}_{2}\right\rangle /\left|\mathbf{e}_{1}\right|^{2}$. Obviously, $|w|<u$. 
Let $k \in \mathbb{Z} \cap[1, m]$ be fixed. For any $n \in \mathbb{N}$, let $h_{k, n}:[0, I] \rightarrow \mathbb{R}$ be defined by

$$
h_{k, n}(x)=\left|(n+x) \mathbf{e}_{1}+k \mathbf{e}_{2}\right|=\left|\mathbf{e}_{1}\right| n f_{k, x}(1 / n),
$$

where $f_{k, x}$ is defined in Lemma 4. That lemma implies that, for all $n \geq 1 / t_{f}$, one has

$$
h_{k, n}(x)=\left|\mathbf{e}_{1}\right| \sum_{i=0}^{\infty} a_{i}(k, x) n^{1-i} .
$$

Let $0 \leq l \leq L$ and let $0 \leq x_{0}<\cdots<x_{l} \leq I$ be some integers. Let us consider $\sum_{j=0}^{l} c_{j} h_{k, n}\left(x_{j}\right)$ and choose integer coefficients $c_{j}, 1 \leq j \leq l$, in such a way that the first $l+2$ leading terms in the decomposition with respect to the powers of $n$ disappear. To do so we use (2.11) to get

$$
\sum_{j=0}^{l} c_{j} h_{k, n}\left(x_{j}\right)=\left|\mathbf{e}_{1}\right| \sum_{i=0}^{\infty} n^{1-i} \sum_{j=0}^{l} c_{j} a_{i}\left(k, x_{j}\right) .
$$

Equating the first $l+2$ coefficients to zero, we obtain the linear system

$$
\sum_{j=0}^{l} c_{j} a_{i}\left(k, x_{j}\right)=0, \quad 0 \leq i \leq l+1
$$

of $l+2$ equations in $l+1$ variables. According to Lemma 4, it is equivalent to the linear system

$$
\sum_{j=0}^{l} c_{j} x_{j}^{i}=0, \quad 0 \leq i \leq l-1,
$$

of $l$ equations in $l+1$ variables. Since the system has integer coefficients, it has an integer non-zero solution $c_{j}(x), 0 \leq j \leq l$, where $x=\left(x_{0}, \ldots, x_{l}\right)$. Moreover, since $a_{l+2}(k, \cdot)$ is a polynomial of degree $l$, we have

$$
\sum_{j=0}^{l} c_{j}(x) a_{l+2}\left(k, x_{j}\right)=: C(k, x) \neq 0 .
$$

This implies

$$
\sum_{j=0}^{l} c_{j}(x) h_{k, n}\left(x_{j}\right)=\left|\mathbf{e}_{1}\right| C(k, x) n^{-l-1}+o\left(n^{-l-1}\right) .
$$

Observe that this asymptotics is uniform in $l, x$, and $k$ as they can take only finitely many values. Hence

$$
M_{1} n^{-l-1}<\left|\sum_{j=0}^{l} c_{j}(x) h_{k, n}\left(x_{j}\right)\right|<M_{2} n^{-l-1},
$$


for all $l, x$, and $k$, with some constants $M_{1}, M_{2}>0$.

Step 3. Now we will show that there is $i(\rho) \in \mathbb{Z} \cap[0, I]$ such that $\omega\left[b_{i(\rho)}(\rho) \eta(\rho)\right]>\rho^{-\varepsilon / 3}$.

First, let us choose $l(\rho) \in \mathbb{Z} \cap[0, L]$ in such a way that it satisfies

$$
\rho^{-\varepsilon / 4}<\rho^{-\frac{l(\rho)+1}{m-2}} \eta(\rho)<\rho^{-\varepsilon / 8}
$$

This is equivalent to

$$
\frac{\log \eta(\rho)}{\log \rho}+\varepsilon / 8<\frac{l(\rho)+1}{m-2}<\frac{\log \eta(\rho)}{\log \rho}+\varepsilon / 4 .
$$

By the assumption on $\eta$ and since it is bounded by $\rho$ from above, we have

$$
\liminf _{\rho \rightarrow \infty} \frac{\log \eta(\rho)}{\log \rho} \geq 0 \quad \text { and } \quad \limsup _{\rho \rightarrow \infty} \frac{\log \eta(\rho)}{\log \rho} \leq 1 .
$$

Now the existence of $l(\rho)$ for all $\rho$ large enough follows from $\frac{1}{m-2}<\varepsilon / 8$ and $\frac{L+1}{m-2}=\frac{2 m+1}{m-1}>2>$ $1+\varepsilon / 4$.

Second, since $I=L m+1$, by the pigeonhole principle there are integers $0 \leq x_{0}(\rho)<\cdots<$ $x_{l(\rho)}(\rho) \leq I$ such that all $k_{x_{i}(\rho)}(\rho)$ are equal, $0 \leq i \leq l(\rho)$. Denote the corresponding value by $k(\rho)$. Using uniform bound (2.12), formula (2.9), and estimate (2.13) we obtain

$$
\eta(\rho)\left|\sum_{j=0}^{l(\rho)} c_{j}(x(\rho)) h_{k(\rho), n(\rho)}\left(x_{j}(\rho)\right)\right|>M_{1} n(\rho)^{-l(\rho)-1} \eta(\rho) \asymp \rho^{-\frac{l(\rho)+1}{m-2}} \eta(\rho)>\rho^{-\varepsilon / 4} .
$$

On the other hand, by (2.12)

$$
\eta(\rho)\left|\sum_{j=0}^{l(\rho)} c_{j}(x(\rho)) h_{k(\rho), n(\rho)}\left(x_{j}(\rho)\right)\right|<M_{2} n(\rho)^{-l(\rho)-1} \eta(\rho) \asymp \rho^{-\frac{l(\rho)+1}{m-2}} \eta(\rho)<\rho^{-\varepsilon / 8}<1 / 2
$$

and so

$$
\omega\left[\eta(\rho) \sum_{j=0}^{l(\rho)} c_{j}(x(\rho)) h_{k(\rho), n(\rho)}\left(x_{j}(\rho)\right)\right]>M_{3} \rho^{-\varepsilon / 4}
$$

with some constant $M_{3}>0$ for all $\rho$ large enough.

Third, assume $\omega\left[b_{i}(\rho) \eta(\rho)\right] \leq \rho^{-\varepsilon / 3}$ for all $i \in \mathbb{Z} \cap[0, I]$. For $i \in\left\{x_{0}(\rho), \ldots, x_{l(\rho)}(\rho)\right\}$ we have

$$
\omega\left[h_{k(\rho), n(\rho)}(i) \eta(\rho)\right]=\left[b_{i}(\rho) \eta(\rho)\right] \leq \rho^{-\varepsilon / 3} .
$$

By the triangle inequality and using the fact that all $c_{j}(x)$ are integers we then obtain

$$
\omega\left[\eta(\rho) \sum_{j=0}^{l(\rho)} c_{j}(x(\rho)) h_{k(\rho), n(\rho)}\left(x_{j}(\rho)\right)\right] \leq \sum_{j=0}^{l(\rho)}\left|c_{j}(x(\rho))\right| \omega\left[h_{k(\rho), n(\rho)}\left(x_{j}(\rho)\right) \eta(\rho)\right]<M_{4} \rho^{-\varepsilon / 3},
$$


where $M_{4}=2 \sup _{x} \sum_{j=0}^{l}\left|c_{j}(x)\right|$. It remains to compare this to (2.14) to get a contradiction.

Step 4. Let us now construct an element $\mathbf{b}(\rho)$ with the required properties.

If $\omega\left[b_{i(\rho)}(\rho) \eta(\rho)\right] \geq \alpha$ then we can take $\mathbf{b}(\rho)=\mathbf{b}_{i(\rho)}(\rho)$ since $b_{i(\rho)}(\rho) \leq \rho^{\varepsilon / 3} \leq \rho^{\varepsilon}$ and $\omega\left[4 b_{i(\rho)}(\rho) \rho\right] \geq 8 \alpha \geq 4 \alpha$.

Suppose $\omega\left[b_{i(\rho)}(\rho) \eta(\rho)\right]<\alpha$. Define

$$
q:=\left\lfloor 3 \alpha \omega\left[b_{i(\rho)}(\rho) \eta(\rho)\right]^{-1}\right\rfloor \leq 3 \alpha \rho^{\varepsilon / 3},
$$

where the inequality follows from the Step 3 . Since

$$
q \omega\left[b_{i(\rho)}(\rho) \eta(\rho)\right] \leq 3 \alpha<1 / 2,
$$

we have

$$
\begin{aligned}
\omega\left[q b_{i(\rho)}(\rho) \eta(\rho)\right] & =q \omega\left[b_{i(\rho)}(\rho) \eta(\rho)\right] \\
& \geq\left(3 \alpha \omega\left[b_{i(\rho)}(\rho) \eta(\rho)\right]^{-1}-1\right) \omega\left[b_{i(\rho)}(\rho) \eta(\rho)\right]>3 \alpha-\alpha=2 \alpha .
\end{aligned}
$$

If $\omega\left[4 q b_{i(\rho)}(\rho) \rho\right] \geq 4 \alpha$ then we can take $\mathbf{b}(\rho)=q \mathbf{b}_{i(\rho)}(\rho)$. Indeed, by (2.15) and since $b_{i(\rho)}(\rho)<\rho^{\varepsilon / 3}$ according to Step 1 we have

$$
b(\rho)=q b_{i(\rho)}(\rho) \leq 3 \alpha \rho^{2 \varepsilon / 3} \leq \rho^{\varepsilon}
$$

if $\rho$ is large enough, $\omega[4 b(\rho) \rho] \geq 4 \alpha$ follows from the assumption above, and $\omega[b(\rho) \eta(\rho)] \geq \alpha$ holds by (2.16).

Suppose $\omega\left[4 q b_{i(\rho)}(\rho) \rho\right]<4 \alpha$. Then we take $\mathbf{b}(\rho)=(q-1) \mathbf{b}_{i(\rho)}(\rho)$. The bound $b(\rho) \leq \rho^{\varepsilon}$ holds by the same argument as above. The estimates

$$
\begin{aligned}
\omega[4 b(\rho) \rho] & =\omega\left[4 b_{i(\rho)}(\rho) \rho-4 q b_{i(\rho)}(\rho) \rho\right]>8 \alpha-4 \alpha=4 \alpha \\
\omega[b(\rho) \eta(\rho)] & =\omega\left[q b_{i(\rho)}(\rho) \eta(\rho)-b_{i(\rho)}(\rho) \eta(\rho)\right]>2 \alpha-\alpha=\alpha
\end{aligned}
$$

follow from the triangle inequality, (2.16) and the assumptions above.

\section{Annuli of bounded width}

In this section we consider the case when $\eta$ is bounded away from zero and infinity (although one of the results is proved in a more general setting). It turns out that $\sigma_{1}(\rho, \eta)$ and $\sigma_{2}(\rho, \eta)$ behave differently depending on whether $d \equiv 3 \bmod 4$ or not. In the case $d \not \equiv 3 \bmod 4$ the precise asymptotics is computed in Theorem 7 , as in such dimensions the trigonometric term appearing in the asymptotics of the Fourier coefficients can be easily kept away from zero (see Lemma 6). In the case $d \equiv 3 \bmod 4$ controlling the trigonometric term becomes more difficult (this is done using Lemma 5), which results in an upper and lower bound becoming different and not delivering a precise asymptotics. Moreover, it turns out that such an asymptotics does not exist as the norms behave differently along subsequences. Hence the dimensions $d \equiv 3 \bmod 4$ are critical and we study them in Theorem 8 . 
Lemma 6. Assume $\eta \asymp 1$ and $d \not \equiv 3 \bmod 4$. Then there are positive constants $c_{1}, c_{2}$ such that for all $\rho>0$ there is $\mathbf{b}(\rho) \in \Gamma^{*}$ satisfying $b(\rho)<c_{1}$ and

$$
|\sin (b(\rho) \eta(\rho)) \sin (b(\rho) \rho-\theta)|>c_{2} .
$$

Proof. Let us first prove that there are two vectors $\mathbf{b}_{1}, \mathbf{b}_{2} \in \Gamma^{*}$ such that $b_{1} / b_{2} \notin \mathbb{Q}$. Suppose this is not true. Then, without loss of generality (by rescaling the lattice), we can assume that the numbers $\left|\mathbf{e}_{1}\right|,\left|\mathbf{e}_{2}\right|$, and $\left|\mathbf{e}_{1}+\mathbf{e}_{2}\right|$ are integers. Thus,

$$
\left\langle\mathbf{e}_{1}, \mathbf{e}_{2}\right\rangle=\frac{\left|\mathbf{e}_{1}+\mathbf{e}_{2}\right|^{2}-\left|\mathbf{e}_{1}\right|^{2}-\left|\mathbf{e}_{2}\right|^{2}}{2} \in \mathbb{Z} .
$$

This implies that any $\mathbf{a}(n)=n \mathbf{e}_{1}+\mathbf{e}_{2}$ satisfies $|\mathbf{a}(n)|^{2} \in \mathbb{Z}$. Since $|\mathbf{a}(n)| \in \mathbb{Q}$ this implies $|\mathbf{a}(n)| \in \mathbb{Z}$. On the other hand,

$$
|\mathbf{a}(n)|=\sqrt{n^{2}\left|\mathbf{e}_{1}\right|^{2}+\left|\mathbf{e}_{2}\right|^{2}+2 n\left\langle\mathbf{e}_{1}, \mathbf{e}_{2}\right\rangle}=n\left|\mathbf{e}_{1}\right|+\frac{\left\langle\mathbf{e}_{1}, \mathbf{e}_{2}\right\rangle}{\left|\mathbf{e}_{1}\right|}+\frac{\left|\mathbf{e}_{1}\right|^{2}\left|\mathbf{e}_{2}\right|^{2}-\left\langle\mathbf{e}_{1}, \mathbf{e}_{2}\right\rangle^{2}}{2 n\left|\mathbf{e}_{1}\right|^{3}}+o(1 / n)
$$

as $n \rightarrow \infty$. Since $\mathbf{e}_{1}$ and $\mathbf{e}_{2}$ are not collinear, the coefficient in front if the term of order $1 / n$ is non-zero. This contradicts $|\mathbf{a}(n)|$ being integer for all $n$.

Let now $\mathbf{b}_{1}, \mathbf{b}_{2} \in \Gamma^{*}$ be such that $b_{1} / b_{2} \notin \mathbb{Q}$. We will show that, for any $\rho>0$, we may choose $\mathbf{b}(\rho)$ to be one of the four points $\mathbf{b}_{1}, \mathbf{b}_{2}, 2 \mathbf{b}_{1}, 2 \mathbf{b}_{2}$, so we choose $c_{1}$ to be larger than $\max \left\{2 b_{1}, 2 b_{2}\right\}$.

Since $b_{1} / b_{2} \notin \mathbb{Q}$, we have

$$
\left|\sin \left(2 b_{1} x\right)\right|+\left|\sin \left(2 b_{2} x\right)\right| \neq 0 \quad \text { for all } x \neq 0 .
$$

As $\eta$ takes values in a compact interval not containing zero, there is a constant $\hat{c}_{1}>0$ such that

$$
\left|\sin \left(2 b_{1} \eta(\rho)\right)\right|+\left|\sin \left(2 b_{2} \eta(\rho)\right)\right|>\hat{c}_{1} \quad \text { for all } \rho>0 .
$$

Hence for any $\rho$, there is $i(\rho) \in\{1,2\}$ such that

$$
\left|\sin \left(2 b_{i(\rho)} \eta(\rho)\right)\right|>\hat{c}_{1} / 2 \text {. }
$$

Using the double angle formula, we obtain

$$
\left|\sin \left(b_{i(\rho)} \eta(\rho)\right)\right|>\hat{c}_{1} / 4 .
$$

On the other hand, since $\theta \neq \pi m$, we have

$$
|\sin (x-\theta)|+|\sin (2 x-\theta)| \neq 0 \quad \text { for all } x \in \mathbb{R} .
$$

Since this is a continuous periodic function, it is bounded away from zero by a constant $\hat{c}_{2}>0$ and so

$$
\left|\sin \left(b_{i(\rho)} \rho-\theta\right)\right|+\left|\sin \left(2 b_{i(\rho)} \rho-\theta\right)\right|>\hat{c}_{2} .
$$

Hence we have for either $\mathbf{b}(\rho)=\mathbf{b}_{i(\rho)}$ or $\mathbf{b}=2 \mathbf{b}_{i(\rho)}$,

$$
|\sin (b(\rho) \rho-\theta)|>\hat{c}_{2} / 2 .
$$

The result follows with $c_{2}=\hat{c}_{1} \hat{c}_{2} / 8$. 
Theorem 7. Assume $\eta(\rho) \asymp 1$ and $d \not \equiv 3 \bmod 4$. Then

$$
\sigma_{1}(\rho, \eta(\rho)) \asymp \sigma_{2}(\rho, \eta(\rho)) \asymp \rho^{\frac{d-1}{2}} .
$$

Proof. The upper bound follows from Lemma 2. To get the lower bound, observe that $\theta \neq \pi m$ as $d \not \equiv 3 \bmod 4$. Hence for each $\rho$ we can pick $\mathbf{b}=\mathbf{b}(\rho)$ according to Lemma 6 . Then by Lemma 3

$$
\left|\hat{R}_{\rho, \eta(\rho)}(\mathbf{b}(\rho))\right|>c \rho^{\frac{d-1}{2}},
$$

since the trigonometric part of the first term on the right hand side of (2.2) is bounded away from zero by Lemma 6 and the second term is then negligible. The lower bound follows now from Lemma 2.

In the following theorem, the condition $\eta \asymp 1$ is replaced by a weaker condition: $\eta(\rho)$ does not have to be separated from zero but should not approach it too fast.

Theorem 8. Assume $\eta$ satisfies (2.7) and is bounded from above, and $d \equiv 3 \bmod 4$.

(1) For any $\delta>0$, there is a positive constants $c$ such that for all $\rho$ sufficiently large

$$
\rho^{\frac{d-1}{2}-\delta}<\sigma_{1}(\rho, \eta(\rho)) \leq \sigma_{2}(\rho, \eta(\rho))<c \rho^{\frac{d-1}{2}} .
$$

(2) There is a sequence $\rho_{n} \rightarrow \infty$ such that

$$
\sigma_{2}\left(\rho_{n}, \eta\left(\rho_{n}\right)\right)=\rho_{n}^{\frac{d-1}{2}}\left(\frac{\log \log \rho_{n}}{\log \rho_{n}}\right)^{\frac{1}{2 d}} O(1)=\rho_{n}^{\frac{d-1}{2}} o(1)
$$

that is, $\sigma_{1}(\rho, \eta(\rho)) \nprec \rho^{\frac{d-1}{2}}$ and $\sigma_{2}(\rho, \eta(\rho)) \nprec \rho^{\frac{d-1}{2}}$.

Proof. (1) The upper bound follows from Lemma 2. To get the lower bound, observe that since $d \equiv 3 \bmod 4$ we have $\theta=\pi m$ for some $m \in \mathbb{Z}$. Without loss of generality we assume that $\delta<1$ and let $0<\varepsilon<\frac{2 \delta}{d+1}$. For each $\rho$, we pick $\mathbf{b}=\mathbf{b}(\rho)$ according to Lemma 5. It follows from Lemma 3 that

$$
\begin{aligned}
\hat{R}_{\rho, \eta(\rho)}(\mathbf{b}(\rho)) & =-2 \sqrt{\frac{2}{\pi}}(2 \pi)^{\frac{d}{2}} \rho^{\frac{d-1}{2}} b(\rho)^{-\frac{d+1}{2}}(-1)^{m} \sin (b(\rho) \eta(\rho)) \sin (b(\rho) \rho) \\
& +\eta(\rho) \rho^{\frac{d-3}{2}} b(\rho)^{-\frac{d+1}{2}} O(1) .
\end{aligned}
$$

The trigonometric part of the first term on the right hand side is bounded away from zero by Lemma 5 and the second term is then negligible. This, together with the estimate $b(\rho) \leq \rho^{\varepsilon}$, implies that there is a constant $c_{1}>0$ such that for $\rho$ large enough

$$
\left|\hat{R}_{\rho, \eta(\rho)}(\mathbf{b})\right|>c_{1} \rho^{\frac{d-1}{2}-\varepsilon \frac{d+1}{2}}>\rho^{\frac{d-1}{2}-\delta} .
$$

The lower bound follows now from Lemma 2. 
(2) The existence of such a sequence $\rho_{n}$ follows from the argument in the proof of Theorem 3.1 in [6]. Let $n \in \mathbb{N}$ and $M_{n}=\left\{b /(2 \pi): \mathbf{b} \in \Gamma^{*}, 0<b \leq n\right\}$. Lemma 3.4 from [6] states that for any set of reals $\left\{\alpha_{1}, \ldots, \alpha_{m}\right\}$ and any $Q \in \mathbb{N}$ there are integers $p_{1}, \ldots, p_{m}$ and $q$ with $Q \leq q<Q^{m+1}$ such that $\left|\alpha_{i} q-p_{i}\right|<Q^{-1}$ for all $i$. We apply this lemma to the set $M_{n}$. So for $Q=\sqrt{n}$ there is a natural number $\rho_{n}$ such that

$$
n^{\frac{1}{2}} \leq \rho_{n}<n^{\frac{\# M_{n}+1}{2}}
$$

(by \# we denote the number of elements in the set) and

$$
\left|\sin \left(b \rho_{n}\right)\right| \leq\left|\sin \left(2 \pi n^{-1 / 2}\right)\right| \leq 2 \pi n^{-1 / 2} \quad \text { for all } \mathbf{b} \in \Gamma^{*} \text { with } b \leq n .
$$

Since $\eta(\cdot)$ is bounded from above, it follows from Lemma 3 that there is a constant $c_{1}$ such that

$$
\hat{R}_{\rho, \eta(\rho)}^{2}(\mathbf{b}) \leq c_{1} \rho^{d-1} b^{-d-1} \sin ^{2}(b \rho)+c_{1} \rho^{d-3} b^{-d-1}
$$

for all sufficiently large $\rho$ and all $\mathbf{b} \in \Gamma^{*} \backslash\{0\}$. Using Parseval's identity and (3.2) we obtain, for all $n$ large enough,

$$
\begin{aligned}
\left\|R_{\rho_{n}, \eta\left(\rho_{n}\right)}\right\|_{2}^{2} & =\sum_{\mathbf{b} \in \Gamma^{*}} \hat{R}_{\rho_{n}, \eta\left(\rho_{n}\right)}^{2}(\mathbf{b})=\sum_{\mathbf{b} \in \Gamma^{*}, 0<b \leq n} \hat{R}_{\rho_{n}, \eta\left(\rho_{n}\right)}(\mathbf{b})+\sum_{\mathbf{b} \in \Gamma^{*}, b>n} \hat{R}_{\rho_{n}, \eta\left(\rho_{n}\right)}(\mathbf{b}) \\
& \leq c_{1} \rho_{n}^{d-1} \sum_{\mathbf{b} \in \Gamma^{*}, 0<b \leq n} b^{-d-1} \sin ^{2}\left(b \rho_{n}\right)+c_{1} \rho_{n}^{d-1} \sum_{\mathbf{b} \in \Gamma^{*}, b>n} b^{-d-1}+c_{1} \rho_{n}^{d-3} \sum_{\mathbf{b} \in \Gamma^{*} \backslash\{0\}} b^{-d-1} \\
& \leq c_{2} \rho_{n}^{d-1} n^{-1}+c_{2} \rho_{n}^{d-3} \leq c_{3} \rho_{n}^{d-1} n^{-1},
\end{aligned}
$$

with some positive constants $c_{2}, c_{3}$ since $\rho_{n} \geq \sqrt{n}$ by (3.1).

Finally, we use the second of the inequalities (3.1) and the estimate $\frac{\# M_{n}+1}{2} \leq\left(c_{4} n\right)^{d}$ with some $c_{4}>0$ to obtain

$$
\log \rho_{n} \leq\left(c_{4} n\right)^{d} \log n
$$

for all large $n$. Consider the function $f(x)=c_{4} x(\log x)^{\frac{1}{d}}$. It is easy to see that its inverse satisfies $f^{-1}(y)=c_{4}^{-1} y(\log y)^{-\frac{1}{d}}(1+o(1))$ as $y \rightarrow \infty$. Using (3.4) and the monotonicity of $f$ for large values of the argument we obtain

$$
n \geq f^{-1}\left(\left(\log \rho_{n}\right)^{\frac{1}{d}}\right)=\left(\frac{\log \rho_{n}}{\log \log \rho_{n}}\right)^{\frac{1}{d}} O(1)
$$

for large $n$. Combining this with (3.3), we arrive at

$$
\left\|R_{\rho_{n}, \eta\left(\rho_{n}\right)}\right\|_{2}=\rho_{n}^{\frac{d-1}{2}}\left(\frac{\log \log \rho_{n}}{\log \rho_{n}}\right)^{\frac{1}{2 d}} O(1)
$$

for all large $n$. This completes the proof. 


\section{Annuli of width tending to infinity}

In this section we are mainly interested in the case when $\eta(\rho) \rightarrow \infty$ and $\eta(\rho)=o(\rho)$, although the theorem below is proved for a slightly more general case. It turns out that in that case all dimensions are critical.

Theorem 9. Assume $\lim \sup _{\rho \rightarrow \infty} \eta(\rho)=\infty, \eta(\rho)=o(\rho)$, and $\eta$ satisfies (2.7).

(1) For any $\delta>0$, there is a positive constant c such that for all $\rho$ sufficiently large

$$
\rho^{\frac{d-1}{2}-\delta}<\sigma_{1}(\rho, \eta(\rho)) \leq \sigma_{2}(\rho, \eta(\rho))<c \rho^{\frac{d-1}{2}} .
$$

(2) There is a sequence $\rho_{n} \rightarrow \infty$ such that

$$
\sigma_{2}\left(\rho_{n}, \eta\left(\rho_{n}\right)\right)=\rho_{n}^{\frac{d-1}{2}} o(1)
$$

that is, $\sigma_{1}(\rho, \eta(\rho)) \nsucc \rho^{\frac{d-1}{2}}$ and $\sigma_{2}(\rho, \eta(\rho)) \nsucc \rho^{\frac{d-1}{2}}$.

Proof. (1) The upper bound follows from Lemma 2. To get the lower bound, let $\varepsilon<\frac{2 \delta}{d+1}$ and, for each $\rho$, pick $\mathbf{b}=\mathbf{b}(\rho)$ according to Lemma 5. It follows from Lemma 3 that

$$
\begin{aligned}
\hat{R}_{\rho, \eta(\rho)}(\mathbf{b}(\rho)) & =-2 \sqrt{\frac{2}{\pi}}(2 \pi)^{\frac{d}{2}} \rho^{\frac{d-1}{2}} b(\rho)^{-\frac{d+1}{2}} \sin (b(\rho) \eta(\rho)) \sin (b(\rho) \rho-\theta) \\
& +\eta(\rho) \rho^{\frac{d-3}{2}} b(\rho)^{-\frac{d+1}{2}} O(1) .
\end{aligned}
$$

The trigonometric part of the first term on the right hand side is bounded away from zero by Lemma 5 and the second term is then negligible. This, together with the estimate $b(\rho) \leq \rho^{\varepsilon}$, implies that there is a constant $c_{1}>0$ such that for $\rho$ large enough

$$
\left|\hat{R}_{\rho, \eta(\rho)}(\mathbf{b})\right|>c_{1} \rho^{\frac{d-1}{2}-\varepsilon \frac{d+1}{2}}>\rho^{\frac{d-1}{2}-\delta} .
$$

The lower bound follows now from Lemma 2.

(2) The existence of such a sequence $\rho_{n}$ is proved similarly to Theorem 3.1 in [6]. Let $n \in \mathbb{N}$ and $M_{n}=\left\{b /(2 \pi): \mathbf{b} \in \Gamma^{*}, 0<b \leq n\right\}$. Lemma 3.4 from [6] states that for any set of reals $\left\{\alpha_{1}, \ldots, \alpha_{m}\right\}$ and any $Q \in \mathbb{N}$ there are integers $p_{1}, \ldots, p_{m}$ and $q$ with $Q \leq q<Q^{m+1}$ such that $\left|\alpha_{i} q-p_{i}\right|<Q^{-1}$ for all $i$. We apply this lemma to the set $M_{n}$, choosing the corresponding natural numbers $Q_{n}$ in such a way that $Q_{n} \rightarrow \infty$. Then, for each $n$, there is a natural number $q_{n} \geq Q_{n}$ such that

$$
\left|\sin \left(b q_{n}\right)\right| \leq\left|\sin \left(2 \pi Q_{n}^{-1}\right)\right| \leq 2 \pi Q_{n}^{-1} \quad \text { for all } \mathbf{b} \in \Gamma^{*} \text { with } b \leq n
$$

Since $\eta$ is continuous and $\lim \sup _{\rho \rightarrow \infty} \eta(\rho)=\infty$, for all $n$ large enough there is $\rho_{n}$ such that $\eta\left(\rho_{n}\right)=q_{n}$. Obviously, $\rho_{n} \rightarrow \infty$. 
It follows from Lemma 3 that there is a constant $c_{1}$ such that

$$
\hat{R}_{\rho, \eta(\rho)}^{2}(\mathbf{b}) \leq c_{1} \rho^{d-1} b^{-d-1} \sin ^{2}(b \eta(\rho))+c_{1} \eta(\rho)^{2} \rho^{d-3} b^{-d-1}
$$

for all sufficiently large $\rho$ uniformly in $b \in \Gamma^{*} \backslash\{0\}$. Using Parseval's identity and (4.1) we obtain, for all $n$ large enough,

$$
\begin{aligned}
\left\|R_{\rho_{n}, \eta\left(\rho_{n}\right)}\right\|_{2}^{2} & =\sum_{\mathbf{b} \in \Gamma^{*}} \hat{R}_{\rho_{n}, \eta\left(\rho_{n}\right)}^{2}(\mathbf{b})=\sum_{\mathbf{b} \in \Gamma^{*}, 0<b \leq n} \hat{R}_{\rho_{n}, \eta\left(\rho_{n}\right)}(\mathbf{b})+\sum_{\mathbf{b} \in \Gamma^{*}, b>n} \hat{R}_{\rho_{n}, \eta\left(\rho_{n}\right)}(\mathbf{b}) \\
& \leq c_{1} \rho_{n}^{d-1} \sum_{\mathbf{b} \in \Gamma^{*}, 0<b \leq n} b^{-d-1} \sin ^{2}\left(b \eta\left(\rho_{n}\right)\right) \\
& +c_{1} \rho_{n}^{d-1} \sum_{\mathbf{b} \in \Gamma^{*}, b>n} b^{-d-1}+c_{1} \eta(\rho)^{2} \rho_{n}^{d-3} \sum_{\mathbf{b} \in \Gamma^{*} \backslash\{0\}} b^{-d-1} \\
& \leq c_{2} \rho_{n}^{d-1}\left(Q_{n}^{-2}+n^{-1}+\eta(\rho)^{2} / \rho^{2}\right)=\rho_{n}^{d-1} o(1),
\end{aligned}
$$

with some positive constant $c_{2}$.

\section{Annuli of width tending to zero}

In this section we study the case when $\eta(\rho)$ tends to zero. In this regime, we are only able to deal with $\sigma_{2}(\rho, \eta)$. We prove an upper bound for it in Lemma 10 and then show in Theorem 11 that the dimensions $d \not \equiv 3 \bmod 4$ are non-critical. Then in Theorem 13 we prove that the dimensions $d \equiv 3 \bmod 4$ are critical if $\eta$ goes to zero slowly, but these dimensions are non-critical, if $\eta$ goes to zero sufficiently quickly.

Lemma 10. Assume $\eta(\rho) \rightarrow 0$ as $\rho \rightarrow \infty$. Then there is a positive constant c such that for all $\rho$ large enough

$$
\sigma_{2}(\rho, \eta(\rho))<c \rho^{\frac{d-1}{2}} \eta(\rho)^{\frac{1}{2}} .
$$

Proof. It follows from Lemma 3 that there is a constant $c_{1}$ such that

$$
\hat{R}_{\rho, \eta(\rho)}^{2}(\mathbf{b}) \leq c_{1} \rho^{d-1} b^{-d-1} \sin ^{2}(b \eta(\rho))+c_{1} \eta(\rho)^{2} \rho^{d-3} b^{-d-1} .
$$

Using Parseval's identity and the inequality $|\sin (x)| \leq|x|$ we obtain

$$
\begin{aligned}
\left\|R_{\rho, \eta(\rho)}\right\|_{2}^{2} & =\sum_{\mathbf{b} \in \Gamma^{*}} \hat{R}_{\rho, \eta(\rho)}^{2}(\mathbf{b})=\sum_{\mathbf{b} \in \Gamma^{*}, 0<b \leq 1 / \eta(\rho)} \hat{R}_{\rho, \eta(\rho)}^{2}(\mathbf{b})+\sum_{\mathbf{b} \in \Gamma^{*}, b>1 / \eta(\rho)} \hat{R}_{\rho, \eta(\rho)}^{2}(\mathbf{b}) \\
& \leq c_{1} \rho^{d-1} \eta(\rho)^{2} \sum_{\mathbf{b} \in \Gamma^{*}, 0<b \leq 1 / \eta(\rho)} b^{-d+1}+c_{1} \rho^{d-1} \sum_{\mathbf{b} \in \Gamma^{*}, b>1 / \eta(\rho)} b^{-d-1}+c_{1} \rho^{d-3} \eta(\rho)^{2} \sum_{\mathbf{b} \in \Gamma^{*} \backslash\{0\}} b^{-d-1} \\
& =c_{2} \rho^{d-1} \eta(\rho)+c_{2} \rho^{d-3} \eta(\rho)^{2} .
\end{aligned}
$$

The observation that $\eta(\rho)^{2}=o(\eta(\rho))$ completes the proof. 
Theorem 11. Assume $\eta(\rho) \rightarrow 0$ as $\rho \rightarrow \infty$ and $d \not \equiv 3 \bmod 4$. Then

$$
\sigma_{2}(\rho, \eta(\rho)) \asymp \rho^{\frac{d-1}{2}} \eta(\rho)^{\frac{1}{2}} .
$$

Proof. The upper bound follows from Lemma 10. To prove the lower bound we use the fact that $d \not \equiv 3 \bmod 4$.

It follows from Lemma 3 that

$$
\hat{R}_{\rho, \eta(\rho)}^{2}(\mathbf{b}) \geq \frac{8}{\pi^{2}}(2 \pi)^{d} \rho^{d-1} b^{-d-1} \sin ^{2}(b \eta(\rho)) \sin ^{2}(b \rho-\theta)-\rho^{d-2} b^{-d-1} \eta(\rho) O(1)
$$

uniformly in $\mathbf{b} \in \Gamma^{*} \backslash\{0\}$. For all $\mathbf{b} \in \Gamma^{*} \backslash\{0\}$ satisfying $b<1 / \eta(\rho)$ we have $|\sin (b \eta(\rho))| \geq$ $\frac{1}{2} b \eta(\rho)$. Hence

$$
\hat{R}_{\rho, \eta(\rho)}^{2}(\mathbf{b}) \geq \frac{2}{\pi^{2}}(2 \pi)^{d} \rho^{d-1} b^{-d+1} \eta(\rho)^{2} \sin ^{2}(b \rho-\theta)-\rho^{d-2} b^{-d-1} \eta(\rho) O(1)
$$

uniformly for those $\mathbf{b}$.

Since $d \not \equiv 3 \bmod 4$ we have $\theta \neq \pi m$ for any $m \in \mathbb{Z}$ and so

$$
\sin ^{2}(x-\theta)+\sin ^{2}(2 x-\theta) \neq 0 \quad \text { for all } x \in \mathbb{R} .
$$

Since this is a continuous periodic function, it is bounded away from zero.

Using Parseval's identity we get

$$
\begin{aligned}
& \left\|R_{\rho, \eta(\rho)}\right\|_{2}^{2}=\sum_{\mathbf{b} \in \Gamma^{*}} \hat{R}_{\rho, \eta(\rho)}^{2}(\mathbf{b}) \geq 1 / 2 \sum_{\mathbf{b} \in \Gamma^{*}, 0<b<1 / \eta(\rho)}\left(\hat{R}_{\rho, \eta(\rho)}^{2}(\mathbf{b})+\hat{R}_{\rho, \eta(\rho)}^{2}(2 \mathbf{b})\right) \\
& =\frac{1}{\pi^{2}}(2 \pi)^{d} \rho^{d-1} \eta(\rho)^{2} \sum_{\mathbf{b} \in \Gamma^{*}, 0<b<1 / \eta(\rho)}\left(b^{-d+1} \sin ^{2}(b \rho-\theta)+(2 b)^{-d+1} \sin ^{2}(2 b \rho-\theta)\right)-\rho^{d-2} \eta(\rho) O(1) \\
& \geq 2 \pi^{d-2} \rho^{d-1} \eta(\rho)^{2} \sum_{\mathbf{b} \in \Gamma^{*}, 0<b<1 / \eta(\rho)} b^{-d+1}\left(\sin ^{2}(b \rho-\theta)+\sin ^{2}(2 b \rho-\theta)\right)-o\left(\rho^{d-1} \eta(\rho)\right) \\
& \geq c_{1} \rho^{d-1} \eta(\rho)^{2} \sum_{\mathbf{b} \in \Gamma^{*}, 0<b<1 / \eta(\rho)} b^{-d+1}-o\left(\rho^{d-1} \eta(\rho)\right) \\
& \geq c_{2} \rho^{d-1} \eta(\rho)-o\left(\rho^{d-1} \eta(\rho)\right),
\end{aligned}
$$

which some positive constants $c_{1}, c_{2}$.

For each $\alpha \in(0,1 / 2)$ and $r>0$, denote

$$
\mathcal{B}_{\alpha}(r, \rho)=\left\{\mathbf{a} \in \Gamma^{*}: r<a<2 r \text { and } \omega[a \rho] \geq \alpha\right\} .
$$

Lemma 12. Let $r$ be a function of $\rho$ such that, as $\rho \rightarrow \infty$, either $r(\rho) \rho^{-\gamma} \rightarrow \infty$ for all $\gamma$ or $r(\rho) \asymp \rho^{\gamma}$ for some $\gamma>0$. Then there is $\alpha \in(0,1 / 2)$ and $c \in(0, \pi / 4)$ such that

$$
\# \mathcal{B}_{\alpha}(\operatorname{cr}(\rho), \rho) \asymp r(\rho)^{d} .
$$


Proof. For each $\alpha \in(0,1 / 2), c \in(0, \pi / 4)$ and $m \in \mathbb{N}$, denote

$$
\begin{gathered}
\mathcal{A}_{\alpha, c, m}(\rho)=\left\{\mathbf{a} \in \Gamma^{*}: \operatorname{cr}(\rho)<a<2 c r(\rho) \text { and at least one of the points } \mathbf{a}+k \mathbf{e}_{1}, 0 \leq k \leq m,\right. \\
\text { satisfies } \left.\omega\left[\left|\mathbf{a}+k \mathbf{e}_{1}\right| \rho\right] \geq \alpha\right\} .
\end{gathered}
$$

It is sufficient to show that there are parameters $\alpha \in(0,1 / 2), c \in(0, \pi / 4)$ and $m \in \mathbb{N}$ such that $\# \mathcal{A}_{\alpha, c, m}(\rho) \asymp r(\rho)^{d}$.

Denote $\theta=\frac{\left\langle\mathbf{a}, \mathbf{e}_{1}\right\rangle}{|\mathbf{a}|\left|\mathbf{e}_{1}\right|} \in[-1,1]$. Compute

$$
\left|\mathbf{a}+k \mathbf{e}_{1}\right|=\sqrt{a^{2}+2 k a\left|\mathbf{e}_{1}\right| \theta+k^{2}\left|\mathbf{e}_{1}\right|^{2}}=a g_{\theta}\left(k\left|\mathbf{e}_{1}\right| / a\right)=\sum_{i=0}^{\infty} b_{i}(\theta) k^{i}\left|\mathbf{e}_{1}\right|^{i} a^{1-i},
$$

according to Lemma 4.

Put $m=1$ if $r(\rho) \rho^{-\gamma} \rightarrow \infty$ for all $\gamma>0$ and $1 / \gamma<m \leq 1 / \gamma+1$ if $r(\rho) \asymp \rho^{\gamma}$. Consider

$$
\sum_{k=0}^{m} c_{k}\left|\mathbf{a}+k \mathbf{e}_{1}\right|=\sum_{i=0}^{\infty} a^{1-i} b_{i}(\theta)\left|\mathbf{e}_{1}\right|^{i} \sum_{k=0}^{m} c_{k} k^{i}
$$

and choose the coefficients $c_{k}, 0 \leq k \leq m$, in such a way that the first $m$ leading terms in the decomposition disappear. To do so, we need to solve the linear system $\sum_{k=0}^{m} c_{k} k^{i}=0,0 \leq i \leq$ $m-1$, of $m$ equations in $m+1$ variables. Since the system has integer coefficients it has an integer non-zero solution $c_{k}, 0 \leq k \leq m$. Moreover, since the Wandermonde matrix is non-degenerated, we have

$$
\left|\mathbf{e}_{1}\right|^{m} \sum_{k=0}^{m} c_{k} k^{m}=: C \neq 0
$$

We have

$$
\begin{aligned}
& \rho \sum_{k=0}^{m} c_{k}\left|\mathbf{a}+k \mathbf{e}_{1}\right|=\rho \sum_{i=0}^{\infty} a^{1-i} b_{i}(\theta)\left|\mathbf{e}_{1}\right|^{i} \sum_{k=0}^{m} c_{k} k^{i} \\
& =C \rho a^{1-m} b_{m}(\theta)+O\left(\rho a^{-m}\right)=C \rho a^{1-m} b_{m}(\theta)+o(1),
\end{aligned}
$$

where the remainder estimate is uniform in $\theta$.

Let $\alpha>0$ be such that $\alpha \sum_{k=0}^{m}\left|c_{k}\right|<1 / 8$.

If all points $\mathbf{a}+k \mathbf{e}_{1}, 0 \leq k \leq m$, satisfy $\omega\left[\left|\mathbf{a}+k \mathbf{e}_{1}\right| \rho\right]<\alpha$ then by the triangle inequality and since all $c_{k}$ are integers one has

$$
\omega\left[\rho \sum_{k=0}^{m} c_{k}\left|\mathbf{a}+k \mathbf{e}_{1}\right|\right] \leq \sum_{k=0}^{m}\left|c_{k}\right| \omega\left[\left|\mathbf{a}+k \mathbf{e}_{1}\right| \rho\right]<1 / 8 .
$$

Hence it suffices to show that there is $c \in(0, \pi / 4)$ such that

$$
\#\left\{\mathbf{a} \in \Gamma^{*}: \operatorname{cr}(\rho)<a<2 c r(\rho), \omega\left[\rho \sum_{k=0}^{m} c_{k}\left|\mathbf{a}+k \mathbf{e}_{1}\right|\right] \geq 1 / 8\right\} \asymp r(\rho)^{d},
$$


which, according to (5.1), would follow from

$$
\#\left\{\mathbf{a} \in \Gamma^{*}: \operatorname{cr}(\rho)<a<2 \operatorname{cr}(\rho), \omega\left[C \rho a^{1-m} b_{m}(\theta)\right]>1 / 4\right\} \asymp r(\rho)^{d} .
$$

Obviously, the condition $\omega\left[C \rho a^{1-m} b_{m}(\theta)\right]>1 / 4$ is equivalent to

$$
\pi n+\pi / 4<C \rho a^{1-m} b_{m}(\theta)<\pi n+3 \pi / 4 \quad \text { for some } n \in \mathbb{Z} .
$$

Since $b_{m}$ is a polynomial of degree $m \geq 1$ it is non-constant and so we can assume without loss of generality (as we can reflect it with respect to the coordinate axes, if necessary) that there are intervals $[p, q] \subset[-1,1]$ and $[P, Q] \subset(0, \infty)$ such that $b_{m}:[p, q] \rightarrow[P, Q]$ is an increasing bijection. Denote by $b_{m}^{-1}$ the inverse of $b_{m}$ from $[P, Q]$ to $[p, q]$.

Observe that (5.2) follows from the set of conditions

$$
\begin{aligned}
& \frac{\pi(n+1 / 4) a^{m-1}}{C \rho}>P, \frac{\pi(n+3 / 4) a^{m-1}}{C \rho}<Q, \\
& b_{m}^{-1}\left(\frac{\pi(n+1 / 4) a^{m-1}}{C \rho}\right)<\theta<b_{m}^{-1}\left(\frac{\pi(n+3 / 4) a^{m-1}}{C \rho}\right) .
\end{aligned}
$$

Let us denote by $\mathcal{S}$ the set of points satisfying (5.3). We need to find a lower bound for $\#\left(\mathcal{S} \cap \Gamma^{*}\right)$. Let us also denote by $\tilde{\mathcal{S}}$ the set of points satisfying

$$
\begin{aligned}
& \frac{\pi(n+1 / 4) a^{m-1}}{C \rho}>P, \frac{\pi(n+3 / 4) a^{m-1}}{C \rho}<Q, \\
& b_{m}^{-1}\left(\frac{\pi(n+2 / 5) a^{m-1}}{C \rho}\right)<\theta<b_{m}^{-1}\left(\frac{\pi(n+3 / 5) a^{m-1}}{C \rho}\right) .
\end{aligned}
$$

A little thought shows that $\tilde{\mathcal{S}}$ is a subset of $\mathcal{S}$; moreover, the distance from each point of $\tilde{\mathcal{S}}$ to the complement of $\mathcal{S}$ is at least $\operatorname{diam}\left(\mathcal{O}^{*}\right)$ (for large enough $\rho$ ). Therefore, a standard covering argument implies that

$$
\#\left(\mathcal{S} \cap \Gamma^{*}\right) \leq \frac{\operatorname{vol}(\tilde{\mathcal{S}})}{\operatorname{vol}\left(\mathcal{O}^{*}\right)} .
$$

Now let us estimate the RHS of (5.5). For each $a$ such that $\operatorname{cr}(\rho)<a<2 c r(\rho)$ the number $M(a, \rho)$ of admissible integers $n$ (i.e. integers $n$ satisfying the first two conditions in (5.4)) can be estimated by

$$
M(a, \rho) \geq \pi^{-1}(Q-P) C \rho a^{1-m}-3 / 2 \geq \pi^{-1}(Q-P) C \rho(2 c r(\rho))^{1-m}-3 / 2 .
$$

If $m \neq 1 / \gamma+1$ then we pick any $c \in(0, \pi / 4)$. Since the expression in the right hand side of (5.6) tends to infinity with $\rho$, there is $\kappa_{1}>0$ such that $M(a, \rho)>\kappa_{1} \rho r(\rho)^{1-m}$. If $m=1 / \gamma+1$ we pick $c \in(0, \pi / 4)$ so small that $M(a, \rho) \geq 1$.

Each admissible $n$ gives an interval of values of $\theta$ via the last inequality in (5.4), and by the mean value theorem there is a constant $\kappa_{1}$ (depending only on the polynomial $b_{m}$ ) such that the 
lengths of all those intervals are bounded below by $\kappa_{1} \rho^{-1} a^{m-1}$. Each such interval corresponds to belts of widths greater than $\kappa_{2} \rho^{-1} a^{m} \rightarrow \infty$ (with some $\kappa_{2}>0$ ) on the sphere of radius $a$. The total width of all belts is bounded below by $M(a, \rho) \kappa_{2} \rho^{-1} a^{m}$, and thus is bounded below by $\kappa_{3} r$ for some $\kappa_{3}>0$. This implies that the admissible area of each sphere of radius $a$ is proportional to the area of the sphere, and so the admissible volume of the annulus $\operatorname{cr}(\rho)<a<2 \operatorname{cr}(\rho)$ is proportional to the volume of the whole annulus. Thus,

$$
\operatorname{vol}(\tilde{\mathcal{S}}) \asymp r(\rho)^{d}
$$

which completes the proof.

Theorem 13. Assume $\eta(\rho) \rightarrow 0$ as $\rho \rightarrow \infty$ and $d \equiv 3 \bmod 4$.

(1) If $\eta(\rho) \rho^{\gamma} \rightarrow 0$ for all $\gamma>0$ or $\eta(\rho) \asymp \rho^{-\gamma}$ for some $\gamma>0$ then

$$
\sigma_{2}(\rho, \eta(\rho)) \asymp \rho^{\frac{d-1}{2}} \eta(\rho)^{\frac{1}{2}} .
$$

(2) If $\eta(\rho) \rho^{\gamma} \rightarrow \infty$ for all $\gamma>0$ then for any $\delta>0$ there is a positive constant $c$ such that for all $\rho$ sufficiently large

$$
\rho^{\frac{d-1}{2}-\delta}<\sigma_{2}(\rho, \eta(\rho))<c \rho^{\frac{d-1}{2}} \eta(\rho)^{\frac{1}{2}} .
$$

If

$$
\eta(\rho)\left(\frac{\log \rho}{\log \log \rho}\right)^{\frac{1}{d}} \rightarrow \infty \quad \text { as } \quad \rho \rightarrow \infty
$$

then there is a sequence $\rho_{n} \rightarrow \infty$ such that

$$
\sigma_{2}\left(\rho_{n}, \eta\left(\rho_{n}\right)\right)=\rho_{n}^{\frac{d-1}{2}} \eta\left(\rho_{n}\right)^{\frac{1}{2}} O(1),
$$

that is, $\sigma_{2}(\rho, \eta(\rho)) \nprec \rho^{\frac{d-1}{2}} \eta(\rho)^{\frac{1}{2}}$

Proof. Observe that the upper bound in both statements has been proved in Lemma 10, and the lower bound in (2) has been proved in Theorem 8, as its assumption (2.7) is satisfied. So it suffices to prove the lower bound in (1) and construct a sequence $\rho_{n}$.

(1) Since $d \equiv 3 \bmod 4$ we have $\theta=\pi m$ for some $m \in \mathbb{Z}$. It follows from Lemma 3 that, for some constants $c_{1}, c_{2}>0$,

$$
\hat{R}_{\rho, \eta(\rho)}^{2}(\mathbf{b}) \geq c_{1} \rho^{d-1} b^{-d-1} \sin ^{2}(b \eta(\rho)) \sin ^{2}(b \rho)-c_{2} \rho^{d-2} b^{-d-1} \eta(\rho)
$$

uniformly in $\mathbf{b} \in \Gamma^{*} \backslash\{0\}$.

Let $r(\rho)=1 / \eta(\rho)$. By Lemma 12 there is $\alpha \in(0,1 / 2)$ and $c_{3} \in(0, \pi / 4)$ such that $\left|\mathcal{B}_{\alpha}\left(c_{3} r(\rho), \rho\right)\right| \asymp r(\rho)^{d}$. Observe that for any $\mathbf{b} \in \mathcal{B}_{\alpha}\left(c_{3} r(\rho), \rho\right)$ we have $0<c_{3} \leq b \eta(\rho) \leq$ 
$2 c_{3}<\pi / 2$ and so $\sin (b \eta(\rho)) \geq \frac{2}{\pi} b \eta$. Further, any $\mathbf{b} \in \mathcal{B}_{\alpha}\left(c_{3} r(\rho), \rho\right)$ satisfies $\omega[b \rho] \geq \alpha$ and so $\sin ^{2}(b \rho) \geq c_{4}$ for some $c_{4}>0$. Using Parseval's identity we obtain

$$
\begin{aligned}
\left\|R_{\rho, \eta(\rho)}\right\|_{2}^{2} & =\sum_{\mathbf{b} \in \Gamma^{*}} \hat{R}_{\rho, \eta(\rho)}^{2}(\mathbf{b}) \geq \sum_{\mathbf{b} \in \mathcal{B}_{\alpha}\left(c_{3} r(\rho), \rho\right)} \hat{R}_{\rho, \eta(\rho)}^{2}(\mathbf{b}) \\
& \geq c_{5} \rho^{d-1} \eta(\rho)^{2} \sum_{\mathbf{b} \in \mathcal{B}_{\alpha}\left(c_{3} r(\rho), \rho\right)} b^{-d+1}-\rho^{d-2} \eta(\rho) O(1) \\
& \geq c_{6} \rho^{d-1} \eta(\rho)^{2} \sum_{\mathbf{b} \in \Gamma^{*}, c_{3} r(\rho)<b<2 c_{3} r(\rho)} b^{-d+1}-o\left(\rho^{d-1} \eta(\rho)\right) \\
& \geq c_{7} \rho^{d-1} \eta(\rho)^{2} r(\rho)+o\left(\rho^{d-1} \eta(\rho)\right)=c_{7} \rho^{d-1} \eta(\rho)+o\left(\rho^{d-1} \eta(\rho)\right)
\end{aligned}
$$

with some positive constants $c_{5}, c_{6}, c_{7}$.

(2) Consider the sequence $\rho_{n}$ constructed in Theorem 8. Since its assumption (2.7) on $\eta$ is satisfied we have

$$
\left\|R_{\rho_{n}, \eta\left(\rho_{n}\right)}\right\|_{2}=\rho_{n}^{\frac{d-1}{2}}\left(\frac{\log \log \rho_{n}}{\log \rho_{n}}\right)^{\frac{1}{2 d}} O(1)=\rho_{n}^{\frac{d-1}{2}} \eta\left(\rho_{n}\right)^{\frac{1}{2}} O(1)
$$

due to (5.7).

\section{References}

[1] G. E. Andrews, R. Askey, R. Roy. Special Functions. Cambridge University Press, 1999.

[2] Z. Cheng, J. L. Lebowitz, P. Major. On the number of lattice points between two enlarged and randomly shifted copies of an oval. Probab. Theory Rel. Fields, 100 (1994), No. 2, 253-268.

[3] F. Götze. Lattice point problems and values of quadratic forms. Invent. Math., 157 (2004), No. 1, 195-226.

[4] M.N. Huxley. Exponential sums and lattice points. III. Proc. London Math. Soc., (3) 87 (2003), No. 3, 591-609.

[5] D. G. Kendall. On the number of lattice points inside a random oval. Quart. J. Math., Oxford Ser. 19, (1948), 1-26.

[6] L. Parnovski, A. V. Sobolev. On the Bethe-Sommerfeld conjecture for the polyharmonic operator. Duke Math. J., 107 (2001), No. 2, 209-238.

[7] L. Parnovski, A. V. Sobolev. Lattice points, perturbation theory and the periodic polyharmonic operator. Annales H. Poincaré, 2 (2001), 573-581.

[8] M. Skriganov. Geometrical and arithmetical methods in the spectral theory of the multidimensional periodic operators. Proc. Steklov Math. Inst. Vol., (1984) 171. 
[9] A. Walfisz. Gitterpunkte in mehrdimensionalen Kugeln. Warszawa: Panstwowe Wydawnictwo Naukowe, 1957. 